\title{
Some Observations on Resilience and Robustness in Human Systems
}

\author{
Dwight W. Read* \\ Department of Anthropology \\ UCLA \\ Los Angeles, CA 90095 \\ USA \\ email: dread@anthro.ucla.edu
}

\begin{abstract}
Robustness versus resilience of systems is first defined. The definition is applied to the material and cultural adaptive system of the Netsilik Inuit. Three structural conflicts and their resolution through cultural practices are delineated. The importance of system monitoring is discussed, especially with regard to the cultural system.
\end{abstract}

\section{Introduction}

Human systems are complex because humans construct abstract "environments" (cultures) whose impact on behavior is at least as substantial as the material environment. However, as stable (reproducible) regimes may only be achievable at the level of group rather than individual behaviors, some form of groupindividual inter-regulation is necessary.

The concepts of robustness and resilience are applicable to human systems involving cultural as well as physical environments. To the extent that individuals act in accordance with individual interests, group level equilibrium will not be maintained without individuals monitoring the state of the system and modifying behaviors accordingly.

In the first part of this commentary I set out a definition of robustness versus resilience and then I define a resilience index. I next apply these ideas to a group of hunter-gatherers known as the Netsilik Inuit who lived along Hudson Bay in Canada. In addition to their intrinsic interest, Hunter-gatherer societies are of evolutionary importance as they represent the context in which the evolution of Homo sapiens took place.

The Netsilik adaptation to an Arctic environment exemplifies the way in which behavior has both a material and an ideational/cultural dimension. Behavior grounded in material conditions is often found to have optimal or near-optimal configurations that are also relatively stable whereas behavior arising through

\footnotetext{
${ }^{*}$ I thank Sander van der Leeuw and Michael Fischer for their comments on a draft version of this manuscript.
}

cultural conditions is complicated by interplay between group-level benefits and individual interests. Both robustness and resilience play a role in Netsilik adaptation to their physical and cultural environment. Some systems are robust and others are resilient. For behaviors relating to the ideational domain of culture, monitoring of the current state of a sub-system, I conclude, plays a much more prominent role than is true for behaviors arising from material conditions.

\section{Definition of Robustness versus Re- silience}

Consider the wind blowing on a tree. We can think of a tree as a dynamic system in equilibrium with respect to the forces of gravity. Tree growth takes advantage of horizontal symmetry around a vertical axis as a way to distribute gravitational forces so that the downward force due to gravity on one side of the tree is balanced by the downward force of gravity on the other side of the tree.

When the wind blows, the new force acting on the dynamic system of a tree responding to gravity temporarily shifts its current state, as measured by the tree's position in 3-space, to a non-equilibrium configuration and when the wind dies down the tree returns to its equilibrium configuration, just as models of linear systems with stable equilibrium points indicate how a dynamic system responds to a transient impulse. Let us imagine the tree as being represented by a vertical, straight line, $L$, when no wind is blowing and when the wind blows, the line changes its angle, $\alpha$, with respect to the ground while keeping the coordinate location of the line's intersection with the ground fixed. We can measure the horizontal shift, $H$, in the top of the tree when subjected to a wind by the length of the arc subtended by the tip of the line, $L: H=\alpha L$, We will use the magnitude of $H$ as a measure of the extent to which the state of the tree has changed due to the wind.

What differs between trees such as palms that sway in the wind and sycamores that may crash when the wind is too strong is the scale for measuring change in the state of the system due to a transient force. By scale, I mean the scale (meters, centimeters, millime- 
ters, etc.) for a unit of measurement for which the measurement of interest will have a value between 0 and 10 using that unit of measurement. For the palm tree, the scale will be meters whereas for the sycamore tree the scale will perhaps be centimeters for the trunk and main branches of the tree. We also need to specify over what transient states of the system we wish to make a measurement. Here we are interested in a measurement of $H$ for values representing the largest value $H$ may take on before system collapse. Let $F_{M}$ represent the maximum transient force that a system can receive before system collapse. We can use $F_{M}$ to indicate over what force range an appropriate numerical scale for measuring the system's response to a transient force, $F$, should be determined. In the case of trees the magnitude of $F_{M}$ is tree specific and if the transient force, $F$, is such that $F>F_{M}$ then the tree will crash. For our purposes we want to know the response of the tree for $0<<F<F_{M}$; that is, the response over the upper range of forces but below the value, $F_{M}$, for which the system will crash.

In contrast with a sycamore tree, the magnitude of $H$ for a palm tree can be measured using a numerical scale commensurate with a scale for measuring the height of the palm tree. This difference between a palm tree and a sycamore tree provides us with an index for differentiating these two systems using the idea of resilience versus robustness. To do so, let us denote the scale for measuring the magnitude of $H$ by $S_{H}$. Now use the same scale, $S_{H}$, to measure $L$. Let us define a dynamic system as resilient when $H \sim L$ with both measures are based on the scale $S_{H}$ and let us define a dynamic system as robust when $H \ll L$. Let us call $H / L$ the resilience index $(R I)$ for the dynamic system represented by our tree example. For a palm tree RI will differ from the index value for a sycamore tree by at least an order of magnitude.

We can easily generalize this example to any dynamic system for which there is a single quantitative scale appropriate for measuring both the state of the system and for measuring the magnitude of the response of the system to a transient force.

\section{Human Dynamic Systems}

\subsection{Material Conditions versus Idea- tional Conditions}

I will be concerned with two aspects of human societies and how each of these relates to robustness and resilience. The first concerns the manner in which biological systems are analyzed in terms of behavior responding to external conditions where the response pattern is directed by information processed in accordance with the implications of evolution driven by natural selection. Within anthropology this approach has often been called a material approach as it places primary emphasis on the material conditions under which individuals in a human society operate. The second aspect is more controversial in terms of the extent to which it can also be accommodated within standard theories of natural selection driven by repro- ductive fitness (individual and inclusive) and relates to the general topic area referred to as culture, namely the way in which humans respond to internalized systems of meaning that are shared across societal members. This is sometimes referred to as an ideational approach as it is based on shared ideas and concepts, not on external conditions.

Of these two aspects of human societies, systems that can be properly considered under the rubric of material conditions and properties arising through some form of natural selection are less problematic as one finds, empirically, that by and large humans are quite effective in arriving at adaptive/optimal solutions to whatever task is at hand. However, for decision making driven by ideational considerations optimal solutions, it will be argued, are less common and may be dependent on decisions made by other participants, hence greater time/energy is likely to be spent on determining how tasks that relate to ideational considerations should be conducted.

These two extremes of activities that either relate primarily to material conditions or to ideational context provide a modeling simplification that may not be accurate in terms of actual behaviors. Most activities have both a material and an ideational component. Choice of what animal to pursue may be predictable from optimal foraging theory when hunting is done solely for procuring food and/or other animal resources, but hunting may also be done for ideational reasons as well.

\subsection{Resilience and Robustness in a Hunter/Gatherer Society}

\subsubsection{Resource Procurement: Netsilik Inuit}

Consider the yearly round of the Netsilik Inuit, an Inuit group living along Hudson Bay in Canada. ${ }^{1}$ Before the introduction of rifles, the Netsilik followed a pattern of resource procurement that involved three main food resources: salmon in the summer, caribou in the fall and seals in the winter. Spring was a transitional period going from seal hunting to salmon fishing as the season progressed.

Summer salmon fishing was based on small work groups of one or two families distributed over the various tributaries that drained into Hudson Bay. Larger groups would have been less efficient as the extra labor was not needed at the locations where the fishing was done and wider sections of the river would have had too much current for building stone dams across the river.

Fall caribou hunting took advantage of water barriers such as small lakes. The caribou were needed not only for food, but also for their skins that were used for clothing and for tents. The hunting strategy was to force the caribou into the lakes, then to harpoon them from kayaks. Hunting on land would have been inefficient since a herd would disperse once the hunters started to kill the caribou. While caribou hunting as

\footnotetext{
${ }^{1}$ Discussion of the Netsilik is based on [Balikci, 1970].
} 
practiced by the Netsilik was highly effective, it did have the risk that the migratory pattern of the caribou was unpredictable and if the caribou did not follow the anticipated route, caribou would not be obtained and starvation was a likely outcome.

Winter seal hunting used an intriguing solution to hunt seals that, for most of the time, were underwater. As they are mammals, the seals must breathe and so they maintain breathing holes in the pack ice. From the surface it is impossible to determine which breathing hole a seal might use and so the strategy of the Netsilik was to have enough men at seal holes (one hunter per seal hole) to ensure that at least one of the hunters would be at the breathing hole used by a seal. Apparently up to about 20 men were needed to ensure high enough odds of getting enough seals on a regular basis to be able to survive through the winter.

\subsubsection{Optimality of Resource Procurement: Material Conditions}

For our purposes here, we may note that the Netsilik methods of food resource procurement and the size of the labor force involved were close to optimal, if not optimal given the technology available to them. (In the longer version of this paper I sketch out a model for why this should be the case based on finding a local maximum using a hill-climbing algorithm.)

\section{Ideational Framework and Material Conditions}

We now consider the relationships between the material and the ideational conditions of the Netsilik with regard to their traditional way of hunting and living. We begin by characterizing the material model for the Netsilik as indicated in the left side of Figure 1. We now show that three main structural conflicts arose in Netsilik society. The first relates to optimization with respect to material conditions. The second arises as a consequence of the cultural means for implementing a solution to the first structural conflict, and the third arises out of the solution found for the second structural conflict. Taken together, the three solutions made for a resilient system in which parts of the system could be modified in response to transient forces on the system and for which return to "equilibrium conditions" would occur once the transient forces had abated.

\subsection{Structural Conflict 1: Demographic Processes versus an Optimal Con- sumer/Producer Ratio}

From a robustness/resilience viewpoint, we may note that changing the Producer/Consumer $(\mathrm{P} / \mathrm{C})$ ratio to a value $>$
1.0 would make the resource procurement system more robust for the state of the procurement system measured in terms of calories obtained per day per consumer. With a greater number of producers, more variability in resource availability (transient effects) could be accommodated before there would be food shortages that could lead to starvation since a successful hunter of caribou or seal, for example, provides enough for his family's needs and for several other persons as well.

This leads to the first structural conflict (see box labeled "(1)" in Figure 1) the Netsilik had to address, namely the biological facts of reproduction and its implication for family structure versus the family structure that would provide greater robustness against stochastic variation in resource availability. From the viewpoint of a family as a resource procurement unit, it would be desirable to have at least the first born child be a male since a first-born daughter increases the time period for which there is an increase in the number of consumers without an increase in the number of producers, hence places the family at greater risk with respect to stochastic variation in resource procurement. The "solution" from a material viewpoint would be to practice infanticide against at least the first born child if she is a daughter in order to increase the odds that the oldest child in a family is a male. In addition, if the goal is to increase $\mathrm{P} / \mathrm{C}$, the total number of pregnancies desired by a family must include any daughter lost to infanticide; otherwise the effect would be to increase the number of families with a first-born child is a male but the overall sex ratio, hence the $\mathrm{P} / \mathrm{C}$ ratio, would not be affected. That

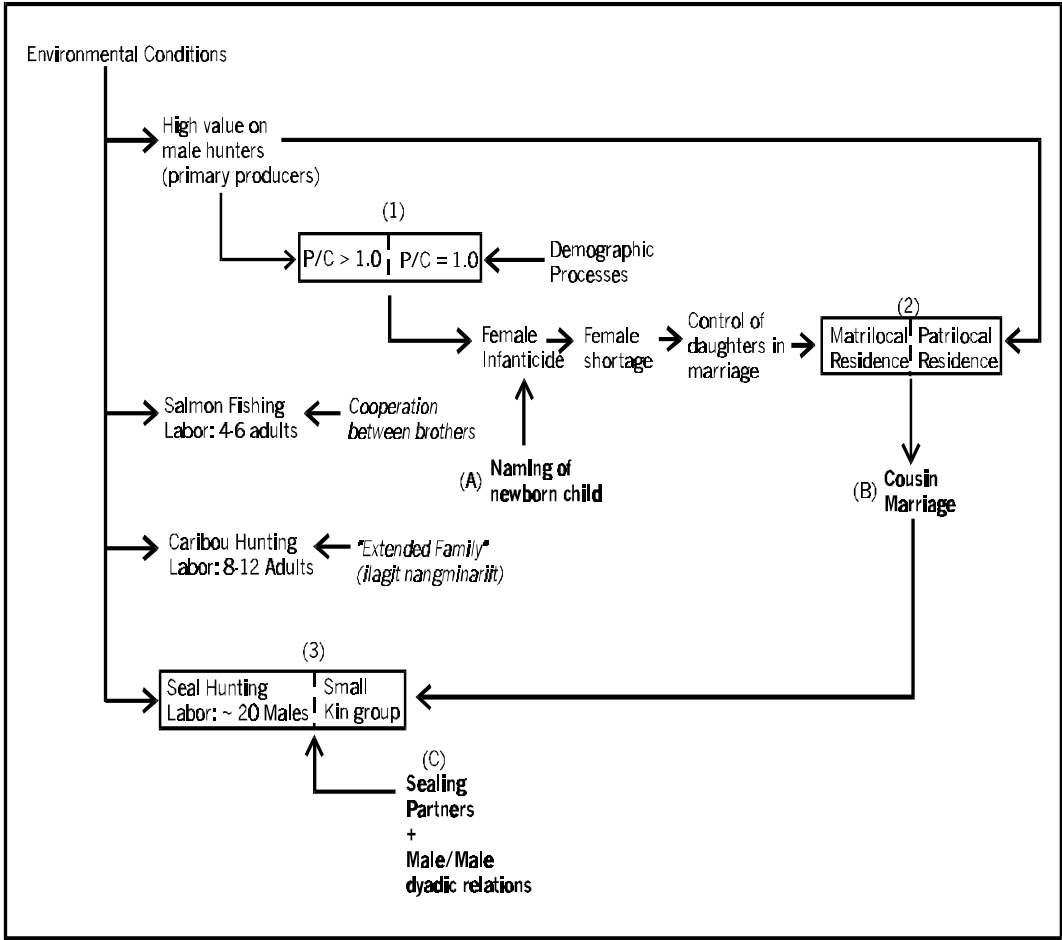

Figure 1 Netsilik adaptation and cultural practices. Boxes identify potential structural conflicts. Bold indicates cultural practices that resolve a potential structural conflict. 
a family's strategy was centered around increasing the $\mathrm{P} / \mathrm{C}$ ratio is born out by census data from when traditional modes of hunting were still being practiced that show a male/female ratio of about 1.5: 1.0.

The means for changing the $\mathrm{P} / \mathrm{C}$ ratio was through infanticide practiced on newborn females. But the Netsilik had to legitimize infanticide. Whether it be via sexual abstinence, abortion or infanticide, human societies seem to require cultural legitimization for behavior that runs counter to maximization of individual fitness. For the Netsilik, legitimization was achieved through a cultural definition that personhood did not accrue to a developing organism until it has been named. The birth-mother names her offspring for one of the spirits that helped her during childbirth, hence infanticide by abandoning a newborn that has not yet been named was not considered murder (see cultural practice labeled "(A)" in Figure 1). An abandoned child could be adopted and the adopting couple, by providing the newborn with a name, gave the newborn its personhood and thereby became its parents. As a consequence, the Netsilik, like other Inuit groups, do not distinguish between biological children and children obtained through adoption. Through a culturally constructed naming practice, the Netsilik provided a "window of opportunity" during which infanticide could occur without it being considered "murder."

If we view the family and its ability to procure needed resources the dynamic system, then the "equilibrium" value from the viewpoint of the adults in the family would be the number of children (taking into account their sexes and ages) for which one is able to provide sufficient food resources throughout the year. A "shock" is a pregnancy as this will increase the number of consumers. One way to return to equilibrium is through determining that the family could obtain sufficient resources for another child and thus deciding to keep the newborn, even if a girl. Alternatively, the family could return to an equilibrium state through infanticide. Unlike a tree that has no control over the shock to the system in the form of wind and must wait for the wind to die down to return to equilibrium, the Netsilik family has the option of removing the shock, hence can control the time frame for return to equilibrium. Is this a robust or a resilient system? If we take the system state, $F$, to be, say, the average number of calories per consumer in the family, then from the viewpoint of a family that decides to keep the child, the system, in the form of a family's means for resource procurement, is robust and $F<F_{M}$ since the additional child is perceived as having only a small, long term effect on the average calories per person in the family. From the perspective of a family that decides upon infanticide, the family's food procurement system is also robust but the additional child is seen as having a significant effect on the family's ability to have sufficient food resources as implied by the decision to practice infanticide. That is, $F>F_{M}$. By practicing infanticide, the family is, in effect, removing a "shock" greater than can be accommodated by the family's food procurement system.

\subsection{Structural Conflict 2: Shortage of Females and Marital Residence}

Female infanticide had long range implications not only for the $\mathrm{P} / \mathrm{C}$ ratio but also for arranging marriages for sons and daughters. Female infanticide leads to a shortage of females available for marriage, hence to placing families with marriageable daughters in an advantageous position with regard to the conditions that would ensue after marriage. We would expect the bride's family to negotiate for the newly married couple to live in the camp of the bride's parents (matrilocal residence) after marriage as this would increase the $\mathrm{P} / \mathrm{C}$ ratio for their camp. However, the groom's family places a high utility on a son as a hunter and they should negotiate for the newly married couple to live with the groom's family after marriage. The two sets of interests are opposed (see box labeled "(2)" in Figure 1) and hence a potential point of conflict that would have to be resolved on a case-by-case basis unless one has culturally specified marriage rules that resolve the potential conflict between the two families involved in the marriage. The cultural rule worked out by the Netsilik is one of cousin marriage (see cultural practice labeled "(B)" in Figure 1). With cousin marriage it was possible to both have patrilocal residence (the likely form of residence when residence groups are based on father/son ties as was true of the Netsilik) and for the bride's parents to gain a producer through the marriage.

Residence groups based on patrilocal residence imply that the residence group is structured around father/son ties. Marriage with a cousin would be with a person already in one's residence group in the case of cousins related through the two respective fathers who are also brothers. For cousins where the parental sibling tie is between a brother and a sister, she may already be a residence in her brother's camp depending on the type of cousin that she married. Lastly, for cousins where the parental sibling tie is between sisters and so their respective husbands need not come from the same residence group, their husbands had a special relationship called angayungoq-nukangor and due to this relation they may, in fact, reside together. In effect, cousin marriage with patrilocal residence had the consequence that the newly married bride and groom were already from the same camp, or at worst were from camps with close kin ties, hence satisfied the concerns of both families with regard to wanting a son or a son-in-law as a hunter.

\subsection{Structural Conflict 3: Limited Kin Networks Due to Cousin Marriage}

But cousin marriage had implications for the breadth of the kinship network of families. Marriages between offspring already closely related as cousins implied that marriage kept kinship networks restricted to a few families. For most food procurement this was not a problem. Salmon fishing only needed two or three families working together and typically would involve brothers. Caribou hunting required a larger work group and the personnel needed for caribou hunting 
could be provided through the residence group based on fathers and sons (a group culturally identified by the Netsilik by the term ilagiit nangminiariit ("own relatives in proper")) (see left side of Figure 1). Seal hunting, however, required a much larger group of males than could be provided through a set of close kin (see box labeled "(3)" in Figure 1).

The problem faced by the Netsilik (and faced by other groups as well) was the difficulty in ensuring cooperation by families under extreme conditions where lack of close kin relations meant that one family had few or no means of coercion to ensure that other families would share resources in an equitable manner. Close kin relations, it should be noted, need not be biologically close kin; rather, kinship and closeness of kin is determined through a culturally constructed system of kinship calculation based on what anthropologists call a kinship terminology, namely the terms the members of a group use for indicating the kind of kin relationship one person has to another. The kinship terminology is a cultural construct based on a "grammar" for how a conceptual structure with the structural features of a terminology, such as the kin relations of one person to another being translatable into the kin relations of a third person to the first two persons [Read and Behrens 19; Read 2001]. That is, and using the American Kinship Terminology as an example, if $\mathrm{B}$ is, say, the Brother of $\mathrm{A}$, and $\mathrm{C}$ is related to $\mathrm{B}$ as, say, the Uncle of $\mathrm{B}$, then the relationship of $\mathrm{C}$ to A (in this case, Uncle) can be calculated from knowing the relationship of $\mathrm{A}$ to $\mathrm{B}$ and the relationship of $\mathrm{C}$ to $\mathrm{B}$.

The problem faced by the Netsilik with regard to strangers is well expressed by an informant' comment to the explorer Rasmussen [1931]: "When they broke camp in his grandfather's day ... the snow knife was never released from the hand... A man in the procession could not stop to make water without great risk, for the one who walked in front might easily get the idea that that man for some reason or other would strike him down from behind, and this suspicion alone might be a sufficient cause of bloodshed.... So it is no wonder that they were doubly cautious when meeting strangers" [as quoted in Balikci, 1970]. Strangers in groups such as the Netsilik are individuals with whom one does not have a kin relation. The Netsilik solved the problem of how to ensure that the men making up the work group for winter sealing be close relations of one another, despite a marriage system that acted against having a large group of close kin, by constructing a system of twelve sealing partners that defined a relation between each pair of males in the hunting group according to the part of seals that would be shared by a hunter with his sealing partners (see cultural practice labeled "(C)" in Figure 1). The sealing partners were selected by the man's mother at the time of his birth and were males who were not already close relatives of the male child. Of the twelve partners, six were flexible in that other males could temporarily take the place of one of these partners, depending on the circumstances of how winter seal hunting groups were being formed.
The utility being maximized via the sealing partners is not quantity of seal meat but equitable and noncontentious access to a resource obtained through chance and for which no single person is likely to obtain a sufficient amount based on his efforts alone. The Netsilik method for equitable distribution is to place the hunter in the position of being the conduit through which the seal is obtained (the hunter stands quietly for hours in the cold of the Arctic winter at a sealing hole) but to remove him from the picture once he has killed a seal. The seal is divided by his wife using a set pattern for the butchering of a seal and the parts of the seal go to the wives of his sealing partners according to the sealing partners established for him by his mother at the time of his birth.

\section{Resilience and Robustness through System Monitoring}

The system of sealing partners made possible the cooperation that was required for a group to survive through the Arctic Winter. From the viewpoint of the particular males residing in a winter sealing camp, the system was resilient as it could accommodate "shocks" in the form of events that prevented the same males from living together in the same sealing camp each year. The system could accommodate other demands that might affect which sealing camp a man and his family might join from one winter to the next. And the system of sealing partners would revert back to the permanent sealing partners in future years. Yet from the viewpoint of the amount of seal meat/ day/ consumer the sealing camp was relatively robust as the number of hunters was large enough to ensure that, most of the time, adequate seal meat would be obtained through the strategy of placing males at the breathing holes of the seals.

Resilience in terms of having the means to vary the personnel making up the winter sealing camp appears to have been a deliberate strategy aimed at ensuring robustness in terms of food availability per consumer. Robustness with respect to individual food needs depended on sharing that ensured each person had equal probability of access to a resource, especially resources stochastic with respect to the likelihood of hunting success and where the resource came in a single, large bundle. A system of distribution had to transcend the individual interest of a hunter with regard to the distribution of his kill. While a system of reciprocity was necessary for the system to work, the Netsilik also realized that one could not depend on individual self-interest to ensure equitable sharing of a seal, especially when cooperation is needed among individuals for whom cultural notions of cooperation and sharing expressed in terms of the proper behavior of close kin to one another are not applicable due to their system of cousin marriage. In effect, the sealing partner solution had group level benefits to which individuals had access only if individual interests were sufficiently suppressed so as to ensure that individual interests did not override group interests. The sealing partner solution, to put it in terms of group versus in- 
dividual selection, was an example of group selection for which the payoff was the ability to survive along the Arctic Coast, thereby gaining access to resources that were not accessible to other groups living inland further to the south such as the Athabaskan Indians.

The Netsilik cultural and material systems, whether robust or resilient, depended heavily upon monitoring current conditions and making adjustments. Decisions to keep a female offspring were based on a family's assessment of its ability to maintain an adequate supply of food resources with an additional consumer in the family. Monitoring of the family's current and expected future state enabled the Netsilik to make the family's food procurement robust in the face of a "shock" in the form of a new food consumer by either incorporating the new food consumer if feasible or through removal of the "shock" so that the family, viewed as a food procurement system, could return to equilibrium. Similarly, parents arranged marriages for marriageable offspring based on the assessment by the respective families of the benefits that would accrue from the proposed marriage. And the system of sealing partners was probably the most complicated of these three systems as it interfaced with a number of aspects of Netsilik life. At one level it had to do with access to seals during the winter (a material utility); at another level it had to do with the problem faced by all societies of constructing social groups in which individual interests are submerged for the benefit of group cohesiveness (an ideational utility). The latter involves monitoring based on assessment and reassessment of relations among individuals and among families and never seems to reach a stable equilibrium.

\section{Conclusion}

A distinction needs to be made between robustness and resilience of a system through the way the system responds to transient impulses. Human systems are complex due to the ability of humans not only to react and respond to their environment, but to construct abstract/symbolically based "environments" - cultures that are as real in terms of their affect on behavior as the material environment in which individuals are embedded. While the concepts of robustness and resilience are also applicable to systems involving these cultural environments, system monitoring appears to take on greater significance, especially in the social domain, as equilibrium may be at the level of group behavior rather than individual behavior. To the extent that individuals act in accordance with individual interests, group level equilibrium will not be maintained without monitoring of the state of the system and modifying behavior accordingly.

The Netsilik Inuit are a good example of the complexities that arise with human systems. Extreme environmental conditions constrain the range of possible activities consistent with surviving Arctic winters and the time sequence and range of resources that must be exploited during a yearly cycle. Netsilik hunting and foraging is optimal or near optimal given the means of food procurement available to them. However, their adaptive behavior depended upon constructing the cultural conditions under which it could occur, beginning with a cultural means for legitimizing female infanticide. But behavior adaptive in one area could also be "maladaptive" in other respects. Female infanticide led to shortage of females, hence to potential conflict by families over who had social control over the groom as a food provider. Their cultural solution emphasized cousin marriage, thereby creating a problem of limited kin networks. That problem was resolved through a system of sealing partners that also removed uncertainty from the distribution of seal meat.

Whereas the behaviors arising from material conditions were stable, behaviors relating to the ideational domain were not and required repeated and frequent monitoring by individuals in terms of their position vis-à-vis other individuals. The Netsilik were engaged in an ongoing, changing system of individual and group relations that depended upon repeated monitoring for maintaining equilibrium. Ideational models need to be monitored through interaction of individuals to keep coherency at the group level. Interaction leads people to do what is required to make an ideational model work in the real world, including behaviors one might not personally induce. Group-level behavior that arises through individuals attempting to instantiate shared ideational models - in a manner that is individually deducible - provides group-level beneficial to individuals. ${ }^{2}$ This is true both of systems that are robust and of systems that are resilient.

\section{References}

Asen Balikci, The Netsilik Eskimo. The Natural History Press, New York, 1970.

Knud Rasmussen, The Netsilik Eskimos. Reports of the Fifth Thule Expedition, Vol. VIII. Copenhagen, 1931.

Dwight Read, What Is kinship? In R. Feinberg and M. Ottenheimer, editors, The Cultural Analysis of Kinship: The Legacy of David Schneider and Its Implications for Anthropological Relativism, University of Illinois Press, Urbana, 2001.

Dwight Read and Clifford Behrens, KAES: An expert system for the algebraic analysis of kinship terminologies, Journal of Quantitative Anthropology 2: 353-93, 1990.

2 Michael Fischer, extends the argument: "the capacity of humans to socially construct their world actually requires strong organizational principles of the ideational system. Otherwise the irregularity of instantiation would tend to degrade the 'shared' aspect of the ideational system as these innovations make their way back into the system. Not to say this doesn't happen, but the ideational component would be unable to reproduce [itself] if many of these innovations were to survive. On the other hand, this also provides a means of incorporating innovations that work sufficiently well and are consistent with the original, or require relatively little transformation of the original, such that others can incorporate them"[personal communication, 2004]. 